\title{
FPGA-Based Time-Domain Channel Estimation in Gaussian Mixture Model
}

\author{
Muhammad Khalid (D), ${ }^{1}$ Abid Muhammad Khan $\left(\mathbb{D},{ }^{2}\right.$ Muhammad Rauf $\left(\mathbb{D},{ }^{3}\right.$ \\ Muhammad Taha Jilani $\left(\mathbb{D},{ }^{4}\right.$ and Sheraz Afzal $\mathbb{D D}^{2}$ \\ ${ }^{1}$ Electrical Engineering Department, Bahria University, Karachi Campus, Karachi, Pakistan \\ ${ }^{2}$ Electronic Engineering Department, Sir Syed University of Engineering and Technology, Karachi, Pakistan \\ ${ }^{3}$ Department of Electronic Engineering, Dawood University of Engineering and Technology, Karachi, Pakistan \\ ${ }^{4}$ Graduate School of Science and Engineering, PAF Karachi Institute of Economics and Technology, Karachi, Pakistan \\ Correspondence should be addressed to Muhammad Khalid; mkhalid.bukc@bahria.edu.pk and Muhammad Rauf; \\ muhammad.rauf@duet.edu.pk
}

Received 4 February 2021; Revised 30 March 2021; Accepted 19 April 2021; Published 3 May 2021

Academic Editor: Dao B. Wang

Copyright (c) 2021 Muhammad Khalid et al. This is an open access article distributed under the Creative Commons Attribution License, which permits unrestricted use, distribution, and reproduction in any medium, provided the original work is properly cited.

\begin{abstract}
The performance of time-domain channel estimation deteriorates due to the presence of Gaussian mixture model (GMM) noise, which results in high mean squared error (MSE) as a challenging issue. The performance of the estimator further decreases when the complexity of the estimator is high due to the high convergence rate. In this paper, an optimized channel estimation method is proposed with low complexity and high accuracy in the GMM environment. In this channel estimation, an improved Gauss-Seidel iterative method is utilized with a minimum number of iterations. The convergence rate of the Gauss-Seidel method is improved by estimating an appropriate initial guess value when no guard bands are used in the orthogonal frequency-division multiplexing (OFDM) symbol. Simulation results provide an acceptable MSE for GMM environments, up to the probability of 5\% impulsive noise component. This paper also presents the design and implementation of the proposed estimator in the NEXYS-2 FPGA platform that provides resources allocation, reconfigurability, schematic, and the timing diagram for detailed insight.
\end{abstract}

\section{Introduction}

In wireless communication, the performance of the system is often limited because it undergoes many unfavorable effects when the signal is transmitted to the receiver. The transmitted signals are normally scattered and reflected and arrived at the receiver through multiple paths [1]. Accordingly, this time-varying channel poses a serious challenge when it comes to estimating it in an efficient manner using the least complex method. Thus, the estimation of the channel is one of the most challenging and core issues in wireless communication. The additive white Gaussian noise (AWGN) has always been the dominant noise model in wireless communication systems, mainly because of two reasons: the first reason is that analytical manipulation is simple and the second one is due to central limit theorem. However, the presence of the impulsive nature of noise in some communication channels gives rise to non-Gaussian characteristics. In general, impulsive noise occurs randomly in the form of a sharp increase in the magnitude [2].

Research efforts, exploited for channel estimation techniques, are with the assumption of AWGN noise models $[3,4]$. It is well known that this assumption is appropriate for many applications but some practical environments exist, which are incorrectly modelled by the AWGN noise model [5]. When the noise deviates from Gaussian, the performance of the existing channel estimators may deteriorate significantly because Gaussian-based estimators are sensitive to the impulsive noise [6]. The purpose of this paper is to develop a robust channel estimator that could perform well in the Gaussian noise environment, as well as in the GMM environment. The non-Gaussian noise is modelled by the well-known GMM model. This noise model characterizes a broad class of non-Gaussian noise distributions that include 
mainly the impulsive noise which is present in several wireless communication systems [7].

Previous studies [8-10] discuss the simplicity and robustness of the least mean square (LMS) and minimum mean square error (MMSE) algorithms based on the AWGN model. However, the performance of these estimators deteriorates due to interpolation error when the density of the pilots is decreased. Furthermore, some more renowned techniques are available in the literature, in which DVB-T2 systems are discussed. The OFDM-based second-generation terrestrial standard DVB-T2 is considered as a flexible approach for system parameters of the mobile and portable television broadcasting. The performance of DVB-T/T2 systems is investigated in [11] and compared with two mobile channel models such as VU30 (Vehicular Urban) and MR100 (Motorway Rural). In order to reduce the energy consumption and implementation complexity, a new standard is defined as DVB-T2-Lite. In paper [12], the performance of DVB-T2 is investigated and compared with DVB-T2-Lite for mobile receivers in typical urban (TU6) mobile channel. It is reported that at new lower values of code rate DVB-T2-Lite outperforms DVB-T2. The performance of the above-discussed estimators is limited by the presence of impulsive noise in a wireless communication system.

To address the impulsive nature of noise, transform domain methods are preferred. Therefore, the authors of papers [13-15] present that the transform domain approach can be helpful to recognize the variations in channel impulse response (CIR). However, the transformed domain channel estimators have relatively large computational complexity $[16,17]$. First and foremost, it requires DFT and then it is followed by zero padding and IDFT operation. Besides, it requires knowledge of channel length for zero padding, which is normally not known to the receiver.

The authors in [18] presented the GMM model and GMM including censored and dropped observation (CDGMM) for improving the performance of the Wi-Fi fingerprinting based indoor positioning system and GMM parameters are estimated. To deal with the censored and dropped data the combination of Bayesian Information Criterion (BIC) to estimate the best number of components of the CD-GMM and EM algorithm is used. The authors achieved improved positioning with the least computational cost; however, the hardware of the proposed estimator is not presented. Literature also reveals some popular state-of-theart channel estimators based on the Bayesian learning technique [19-21]. Another state-of-the-art method is the iterative-based technique [22-28]. However, Bayesian learning techniques are complex and require prior knowledge of the initial values of estimated parameters. Similarly, the iterative based techniques suffer from high complexity problem due to high convergence rate. Consequently, these methods need a large number of hardware resources.

Hence, it is more fruitful to design a channel estimator with low convergence rate and it does not depend upon the density of the pilots in GMM environment. Accordingly, the proposed work focuses on time-domain channel estimation method, which is based on iterative technique that utilizes cyclic cross-correlation between received signal and no guard band subpilot sequence to estimate the channel impulse response. The convergence rate of the Gauss-Seidel method can be improved by selecting an appropriate value as an initial guess.

The main contributions to this article are summarized as follows:

(1) The time-domain channel estimator is presented, which utilizes an appropriate initial guess and improves the convergence rate of iterative Gauss-Seidel method in the GMM environment

(2) The FPGA implementation of the proposed channel estimation is investigated with its hardware resource requirements

(3) Simulation results provide an acceptable MSE, up to the probability of $5 \%$ impulsive noise component in GMM environment

The remainder of the manuscript is organized as follows: Section 2 discusses the system model. Section 3 presents the detailed discussion of an improved Gauss-Seidel method. Simulation parameters and performance evaluation are explained in Section 4. Section 5 covers the FPGA implementation of the proposed estimator. Section 6 presents the results and discussion. Finally, Section 7 concludes this paper.

\section{System Model}

Consider a baseband equivalent, band-limited system model; Figure 1 shows the OFDM Transceiver Block Diagram. In Figure 1, $D[k]$ and $P[k]$ are the frequency-domain signals, represented as follows:

$$
P[k]=\sum_{N=0}^{N_{F F T}-1} p(n) e^{\left(-j 2 \pi k n / N_{F F T}\right)}, D[k]=\sum_{N=0}^{N_{F F T}-1} d(n) e^{\left(-j 2 \pi k n / N_{F F T}\right)},
$$

where $n$ is the time-domain index; $n=0,1, \ldots, N_{F F T}-1$ of an OFDM symbol. To reduce the correlation error in timedomain channel estimation, $P[k]$ is obtained as follows:

$$
P[k]=P_{1}[k]+P_{2}[k] .
$$

The frequency-domain signal $S[k]$ is the sum of pilot and data signal, given as

$$
S[k]=P[k]+D[k] .
$$

After the IFFT operation and adding of cyclic prefix, the signal $s^{\prime}(n)$ is transmitted through the multipath fading channel $h(n)$, represented as

$$
h(n, \tau)=\sum_{i=0}^{L-1} h_{i} e^{j\left(2 \pi / N_{F F T}\right) T n} \delta\left(\tau-\tau_{i}\right),
$$

where $h_{i}$ is the $i^{\text {th }}$ complex path gain, $L$ is total number of channel taps, and $\tau_{i}$ is the normalized path delay. Without loss of generality, it is possible to use the low-pass system 


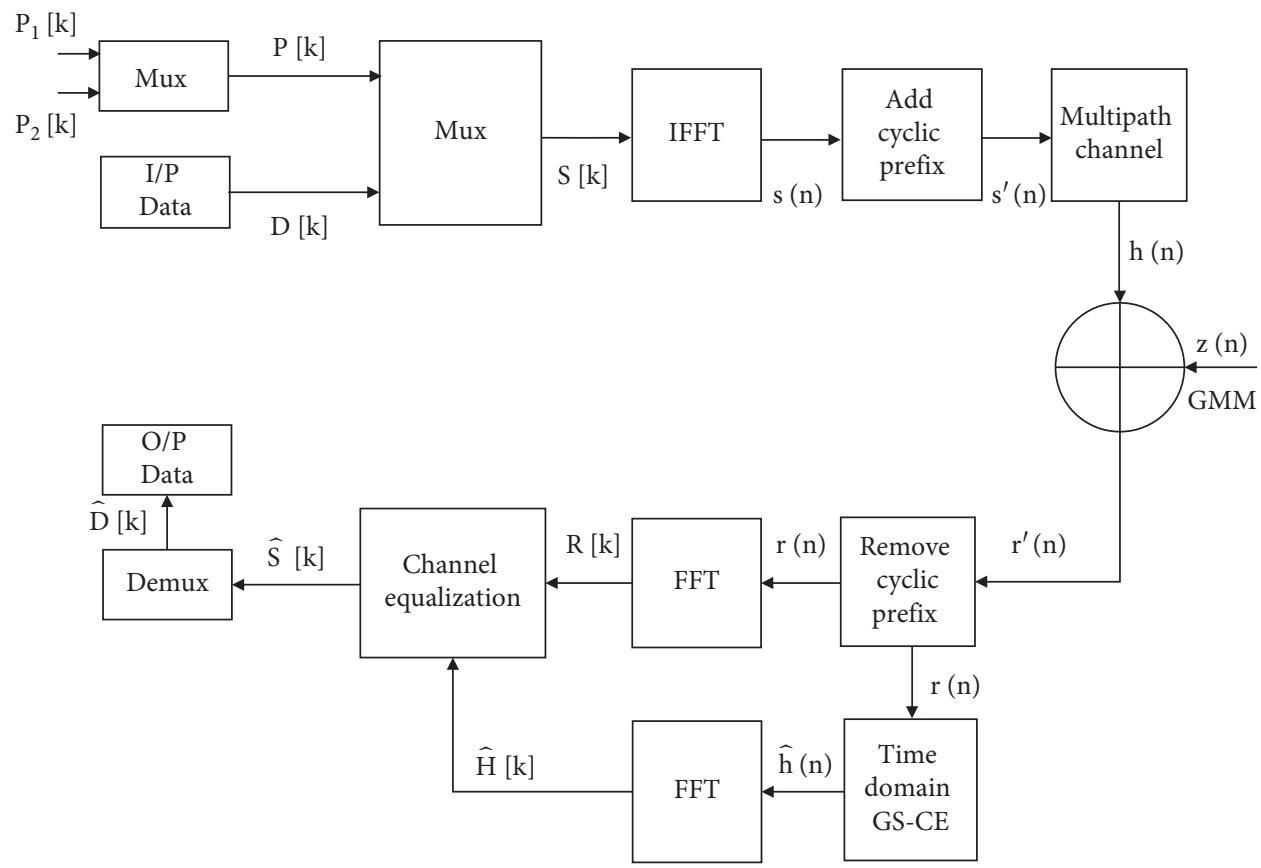

Figure 1: OFDM Transceiver Block Diagram.

model. Thus, the received signal $r(n)$ excluding cyclic prefix can be written as [29]

$$
r(n)=\sum_{m=0}^{N_{F F T}-1} h(m)\left[\mathrm{d}(n-m)_{N_{F F T}}+p(n-m)_{N_{F F T}}\right]+z(n),
$$

where $m$ is the indexing, which is used in time domain, $(.)_{N_{F F T}}$ represents the modulo of $N_{F F T}$, and $z(n)$ represents the GMM noise [19], given as

$$
p(z(n))=(1-\varnothing) \cdot \mathscr{C} \mathcal{N}\left(0, \sigma_{n}^{2}\right)+\varnothing \cdot \mathscr{C} \cdot \mathcal{N}\left(0, T \sigma_{n}^{2}\right),
$$

where $T \gg 1$ represents impulsive-noise strength and $\mathscr{C} \mathscr{N}\left(0, T \sigma_{n}^{2}\right)$ represents the Gaussian distributions with zero mean and variance $\sigma_{n}^{2}$, and $\varnothing$ is the controlling parameter of the impulsive noise level. According to equation (6), variance of GMM is obtained as

$$
\sigma_{z}^{2}=E\left\{z^{2}(n)\right\}=(1-\varnothing) \sigma_{n}^{2}+\varnothing T \sigma_{n}^{2}
$$

Note that if $\varnothing=0$, then $z(n)$ becomes the AWGN model. The cyclic correlation of received signal $r(n)$ with the locally generated $p_{1}(n)$ is given by

$$
C_{r p_{1}}(n)=h(n) \otimes\left[C_{d p_{1}}(n)+C_{p_{1} p_{1}}(n)+C_{p_{2} p_{1}}(n)\right]+C_{z p_{1}}(n) .
$$

In general, $C_{x y}(n)$ is the cyclic correlation between $x$ and $y$ in equation (8). It is shown in [29] that $C_{\mathrm{d} p_{1}}(n)=0$. Thus, the output of cyclic correlation becomes

$$
C_{r p_{1}}(n)=h(n) \otimes\left[C_{p_{1} p_{1}}(n)+C_{p_{2} p_{1}}(n)\right]+C_{z p_{1}}(n) .
$$

The cross correlation of noise $z(n)$ with $p_{1}(n)$ is $C_{z p_{1}}(l)$, which can be represented as Gaussian mixture noise with zero mean and variance $\sigma_{z}^{2}$. Also $C_{z p_{1}}(l)=z(l)$. Therefore, equation (9) can be expressed as

$$
C_{r p_{1}}(l)=h(l) \otimes\left[C_{p_{1} p_{1}}(l)+C_{p_{2} p_{1}}(l)\right]+z(l), \quad 0 \leq l \leq N_{P}-1 .
$$

It is noted that, over the length of $N_{P}, C_{p_{1} p_{1}}(0)$ and $C_{p_{2} p_{1}}(0)$ act like delta functions. Thus, equation (10) can be rewritten as

$$
C_{r p_{1}}(l)=h(l)\left[C_{p_{1} p_{1}}(0)+C_{p_{2} p_{1}}(0)\right]+\left[\sum_{\substack{n_{1}=0, n_{1} \neq l}}^{L-1} h\left(n_{1}\right) \cdot C_{p_{2} p_{1}}\left(N_{P}+l-n_{1}\right)\right]+z(l) .
$$

To estimate the channel impulse response $h(l)$, equation (11) can be written as 


$$
\begin{aligned}
& \qquad(l)=\frac{\sum_{n_{1}=0,}^{L-1}}{C_{p_{1} p_{1}}(0)+C_{p_{2} p_{1}}(0)}-\frac{C_{p_{1}}(l) \cdot l}{C_{p_{p_{1} p_{1}}}(0)+C_{p_{2} p_{1}}(0)} . \\
& \text { (12) that } h(l) \text { is the difference of } \\
& \widehat{h}_{l}^{(k+2)}=\widehat{h}_{l}^{(k+1)}-\frac{\sum_{n_{1}=0,}^{L-1} \hat{h}_{n_{1}}^{k+1} \cdot C_{p_{2} p_{1}}\left(N_{p}-l-n_{1}\right)-z(l)}{C_{p_{1} p_{1}}(0)+C_{p_{2} p_{1}}(0)} .
\end{aligned}
$$

It is clear from equation (12) that $h(l)$ is the difference of two components. The first component is free from windowing attribute due to having no guard bands and the second component contains windowing attribute. If transmitted OFDM signal does not contain guard band subcarriers, then $h(l)$ can be simplified as

$$
h(l)=\frac{C_{r p_{1}}(l)}{C_{p_{1} p_{1}}(0)+C_{p_{2} p_{1}}(0)},
$$

where equation (13) is termed as initial guess for $h(l)$. However, in most practical systems, the transmitted frequency-domain signal contains guard band subcarriers at their respective positions. Therefore, $h(l)$ contains both components. The most straightforward approach for solving equation (13) for $h(l)$, is by using deconvolution. But the problem with the deconvolution is that not only is it computationally complex but also it requires matrix inversion that can have null values in denominator. Consequently, it increases hardware implementation cost and hence the performance of receiver deteriorates [30]. Due to these reasons, the channel estimation using deconvolution is not practically desirable. Herein, the following method is proposed.

\section{Improved Gauss-Seidel Method}

In this section, channel impulse response is estimated by using equation (12). For this purpose, Gauss-Seidel iterative method is utilized. Moreover, the convergence rate of Gauss-Seidel method is improved by selecting an appropriate initial guess, such as using component which is without windowing attribute. Therefore, we have the following:

(1) The estimated initial guess $\widehat{h}_{l}^{(k)}$ (with no guard bands) is given by

$$
\widehat{h}_{l}^{(k)}=\frac{C_{r p_{1}}(l)}{C_{p_{1} p_{1}}(0)+C_{p_{2} p_{1}}(0)}, \quad \text { where } k=0 .
$$

(2) To estimate the next iterative value $\widehat{h}_{l}^{k+1}$, substitute the values of $\widehat{h}_{l}^{k}$ in equation (12).

$$
\hat{h}_{l}^{(K+1)}=\hat{h}_{l}^{k}-\frac{\sum_{\substack{n_{1}=0, n_{1} \neq l}}^{L-1} \hat{h}_{n_{1}}^{k} \cdot C_{p_{2} p_{1}}\left(N_{p}-l-n_{1}\right)}{C_{p_{1} p_{1}}(0)+C_{p_{2} p_{1}}(0)} .
$$

(3) Similarly, for further iterative value,
(4) After each iteration, it keeps checking whether the difference in error is less than the tolerance.

$$
\frac{\widehat{h}_{l}^{(k+1)}-\widehat{h}_{l}^{(k)}}{\widehat{h}_{l}^{(k+1)}}=\epsilon_{r} \text {. }
$$

(5) Iteration process continues till the difference in errors is not smaller than the tolerance $\epsilon_{r}$.

\section{Performance Evaluation}

This section evaluates the performance of the proposed estimator DVB-T2 system in fixed reception scenario over Rayleigh (RL20) channel model, where MSE and BER are estimated for AWGN and GMM channel environments. In order to estimate the channel estimation alone, a perfect synchronization can be assumed, and a rectangular window is implemented by using $N_{P}$ number of null pilot subcarriers in the frequency domain as a guard band. In OFDM systems, quadrature amplitude modulation (QAM) is used extensively, due to having capability of acquiring higher spectral efficiency by setting suitable constellation size. For simulation, data constellation is chosen to be 4QAM and 16-QAM, while pilot subcarriers are unipolar BPSK modulated and boosted with a factor of $16 / 9$. The average transmitted power of symbol is normalized to 1 . For fairly accurate communication, the range of SNR is selected from $0 \mathrm{~dB}$ to $30 \mathrm{~dB}$ for MSE and from 0 to $25 \mathrm{~dB}$ for BER performance. Simulation is performed 1000 times for each SNR. The simulation parameters are shown in Table 1 .

\section{FPGA Implementation}

This section presents the design and implementation of the proposed channel estimator by employing the GS method in NEXYS-2 FPGA that provides reconfigurability, resource allocation, and the timing diagram. A method of implementation of the FPGA scheme has been presented in a recent study $[32,33]$. The hardware implementation of the proposed estimator is implemented in two phases as shown in Figure 2.

Phase I starts with coding and verification of the proposed estimator in MATLAB 9.2 R2017a. It is then translated 
TABLE 1: Simulation parameters taken from [31].

\begin{tabular}{|c|c|}
\hline Parameters & DVB-terrestrial \\
\hline FFT points $\left(N_{F F T}\right)$ mode & 2048 \\
\hline Symbol duration & 224 us \\
\hline Guard interval $\left(N_{G}\right)$ & $1 / 16,14$ us \\
\hline Pilots spacing $\left(N_{F}\right)$ & 16 \\
\hline Number of pilots $\left(N_{P}\right)$ & 128 \\
\hline Signal constellation $D[k]$ & 4 QAM, 16 QAM \\
\hline Code rate & $1 / 2,1 / 3$ \\
\hline FFT sampling frequency $F_{s}$ & $9.14 \mathrm{MHz}$ \\
\hline Channel bandwidth & $8 \mathrm{MHz}$ \\
\hline $\begin{array}{l}\text { Nonzero channel taps of Rayleigh } \\
\text { fading channel }\end{array}$ & 14 \\
\hline Pilot pattern & $16(3)=48$ \\
\hline Pilot subcarrier & 2 \\
\hline Received signal & 1 \\
\hline
\end{tabular}

into VHDL code by using HDL code generation. In VHDL code generation, the HDL coder assigns a workflow advisor that inquires two files: one is function file (contains the main algorithm) and the second one is test bench file (contains inputs). The workflow advisor first defines the input type and then translates the input values into the fixed point from floating point. Finally, the MATLAB code is translated into HDL code as shown in Figure 2. The estimator is translated into HDL using HDL coder in MATLAB, and then this code is further checked and verified by Xilinx 14.2.

Phase II explains the design process execution of FPGA as shown in Figure 2. In Xilinx, the code endures the four main steps. In the first step, design functionality has been verified and checked through a test bench waveform. Simulation results also help to transform into RTL abstraction which describes the algorithm to its circuit-level concept describing the components at the gate-level abstraction that can be useful in hardware design. If the complex operation at the circuit level is not justified and efficient enough in terms of multipliers and address, then the circuit description is changed and simulations are performed again to ensure that the conceptualized approach does function appropriately. In the second step, the HDL code is synthesized, which provides the translation of the code into a gate-level net-list. Furthermore, the postsynthesis simulation verifies and confirms the design results at the gate and flip-flop level to prove the transparency of gate-level abstraction translation. In the third step, the generated gatelevel abstraction undergoes route and placement procedure. Finally, a programming file is generated, which implements the proposed design on the FPGA board.

Experimental setup of the system is shown in Figure 3. The results are verified on the FPGA NEXY-2 board, in which the following parameters are analyzed.

5.1. Resource Allocation. The computation complexity of the estimator is analyzed by using the mathematical expression. The complex mathematical operation is defined in terms of fixed point generation by using floating point to fixed point conversion in MATLAB HDL coder. This real conversion of complex operation has various levels of complexity when

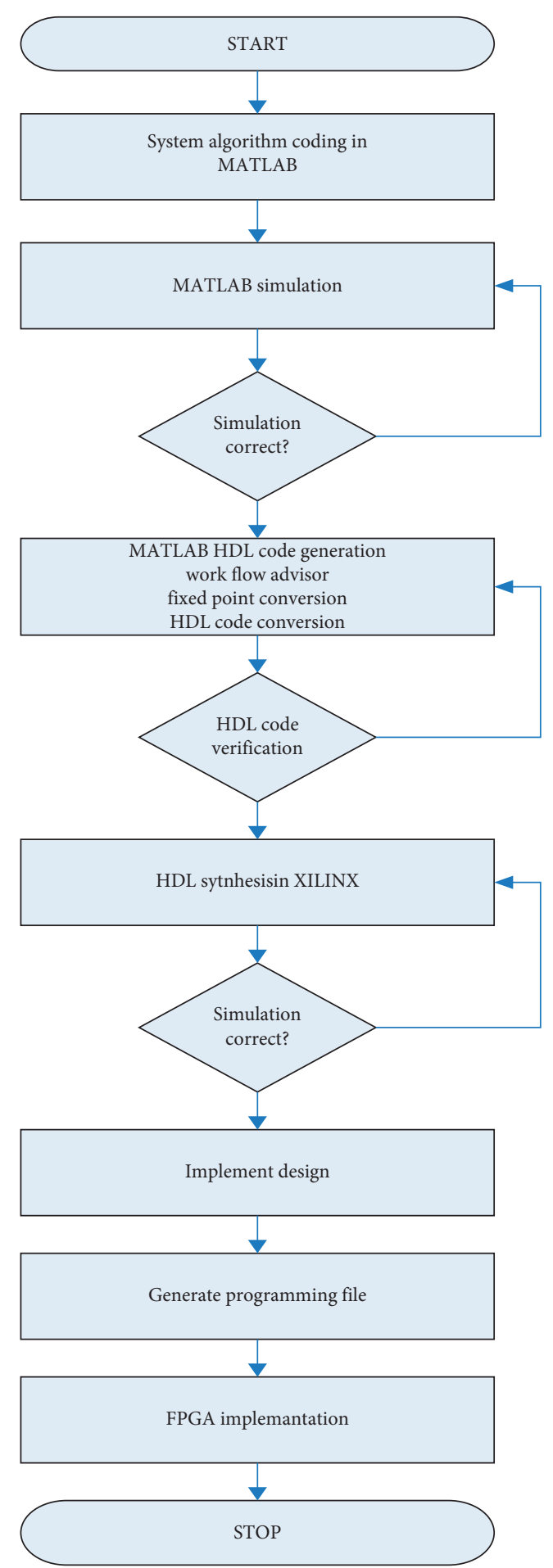

FIgURe 2: Design flow of the system.

defining in terms of hardware design. Table 2 defines the hardware complexity of circuit at gate level of abstraction by using different mathematical operations such as multiplication/division and addition/subtraction.

5.2. Schematic Diagram. Figure 4 shows the schematic diagram for GS estimator, in which three input variable are 


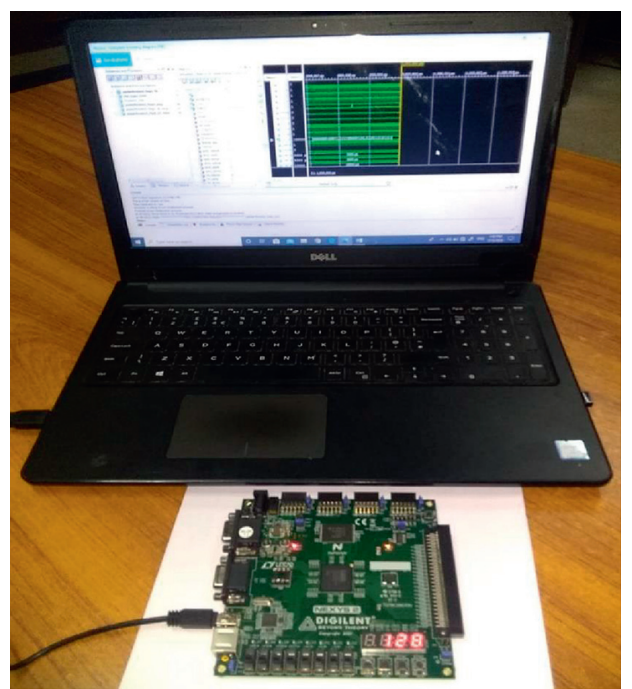

FIGURE 3: Experimental setup on FPGA implementation.

TABLE 2: Hardware recourses allocation.

\begin{tabular}{lc}
\hline Real operations & Operation counts \\
\hline Multiplication/division & 5 \\
Addition/subtraction & 112 \\
\hline
\end{tabular}

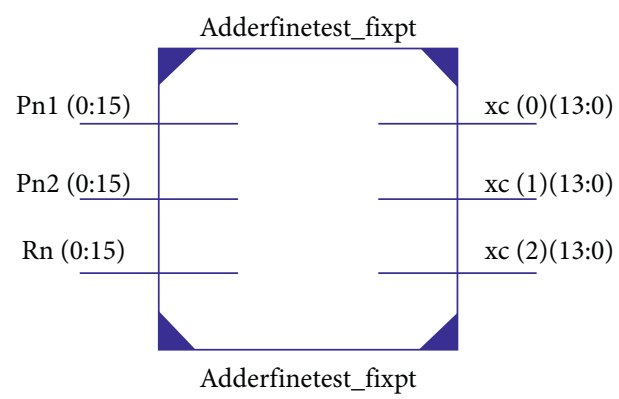

FIgURE 4: Schematic diagram for the GS estimator.

used: two for pilot subcarriers $P_{1}(n), P_{1}(n)$ and one for received signal $R(n)$. However, the three output variables (nonzero channel coefficients) are estimated as shown in Table 1.

5.3. Timing Diagram. Figure 5 represents the timing simulation for the estimator in the GMM environment. It shows the closest emulation that is actually downloaded to device. It allows inspecting all functional and timing requirements for the device. Random bit-oriented input variables for both pilot and received signals are taken on which the outputs are verified.

\section{Results and Discussion}

In this section, first of all, the Gauss-Seidel based timedomain channel estimation method is tested at $13 \mathrm{~dB}$ SNR. Figure 6 depicts the MSE of the CIR, which is plotted against the number of iterations. The legends AWGN pilot Amp. $=1$ and GMM pilot Amp. $=1$ represent the MSE performance with a unity pilot amplitude in AWGN and GMM, respectively. The general behaviour of two legends shows that the MSE converges in one iteration. This is due to the selection of appropriate initial guess as discussed in the last two paragraphs of Section 2. In other words, the larger part of the actual CIR solution is present in an initial guess (no guard bands) term. Therefore, the actual solution of channel estimation is the small correction to the solution with an initial guess. The two legends AWGN pilot Amp. $=2$ and GMM pilot Amp. $=2$ represent the MSE performance with a pilot amplitude of boosted factor 2 . In this case, it is analyzed that the number of iterations increases, and the MSE converges in three iterations as represented by circles. This is due to the higher difference between the correlation error terms arising from $C_{p_{2} p_{1}}(l)_{l \neq 0}$ and $C_{p_{1} p_{1}}(l)+C_{p_{2} p_{1}}(l)_{l=0}$ in equation (12).

Figures 7-9 depict the MSE performance of the proposed channel estimator for the GMM channel with three different values of $\varnothing$ (controlling parameter). Three different values of $\varnothing$ are considered for analyzing the effect of higher probabilities of the impulsive noise component. In Figures 7-9, the MSE performances of existing channel estimators (DFT and MMSE) are compared with the proposed methods (initial guess and GS estimator) with 16-QAM modulation scheme. It is evident that the performance of the GS estimator outperforms those of the other estimators. It can be further seen that the difference of MSE is dominant at the high SNR regime. The comparison of the three figures depicts that the MSE difference between an initial guess and GS estimator reduces at higher values of $\varnothing$. In GMM, there are two error components present in the estimation of CIR. The first one is due to the AWGN and the second one is due to the impulsive noise as discussed in equation (7). At a high value of $\varnothing$, the impulsive noise component is relatively dominant and the MSE of GS estimator is increased.

To observe the effect of the M-order modulation scheme in the GMM environment, the performance of the proposed estimator is evaluated with the 4-QAM in Figures 10 and 11. A similar trend is found with the 4-QAM modulation scheme as observed with 16-QAM. It is also evident that the lower-order modulation scheme improves MSE performance. Furthermore, in the presence of GMM noise, the MSE performance degraded when the value of the controlling parameter $\varnothing$ is increased from 0.01 to 0.05 .

Figures 12-14 show the bit error rate (BER) curves for GMM environments. In BER performance, the convolution code with the code rates of $1 / 2$ and $1 / 3$ is used. The Viterbi algorithm is utilized to decode the convolutional code. Results show that the BER performance of the proposed estimator is improved and a similar trend of MSE is found as discussed in Figures 7-9, respectively.

The low code rate not only increases the error correction capabilities but also increases overhead. The code rate is represented by a number $(k / n)$ and it measures the efficiency of the code. The performance of the proposed estimator is also evaluated with $1 / 3$ code rate in Figures 15 and 16 . Simulation results show that BER decreases with a lower code rate. 


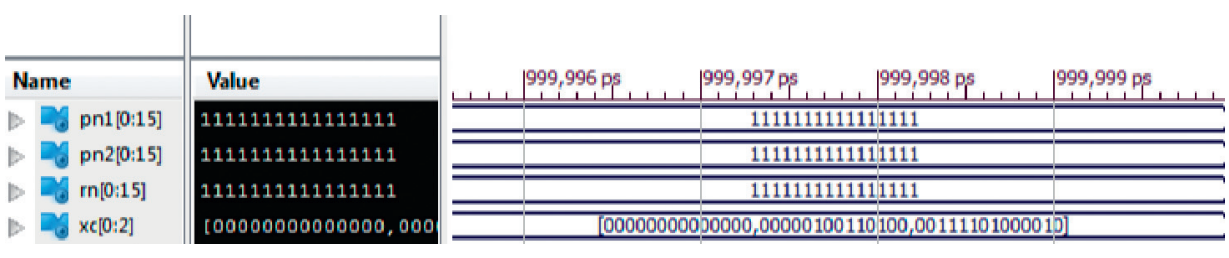

Figure 5: Timing diagram.

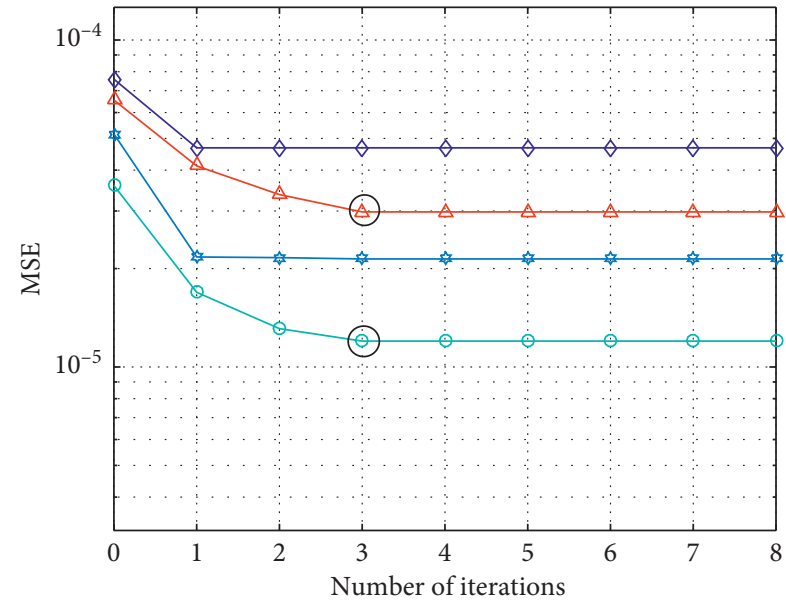

$\diamond$ GMM, pilots amp $=1 \Rightarrow$ AWGN, pilots amp $=1$

$\triangle$ GMM, pilots amp $=2-\odot$ AWGN, pilots amp $=2$

Figure 6: Number of iterations at different pilot's amplitudes.

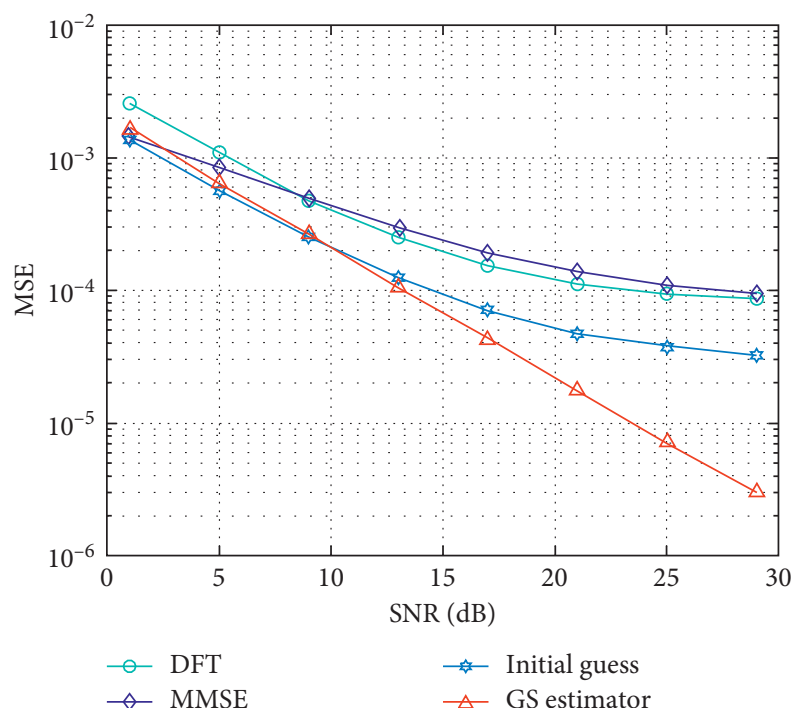

FIgURE 7: Performance of the estimator in AWGN channel with $\varnothing=0, T=0,16-\mathrm{QAM}$.

In Figures 17 and 18, the effects of the boosting factor of the pilot subcarrier for the proposed time-domain Gauss-Seidel based estimator and the conventional frequency-domain MMSE channel estimator are analyzed,

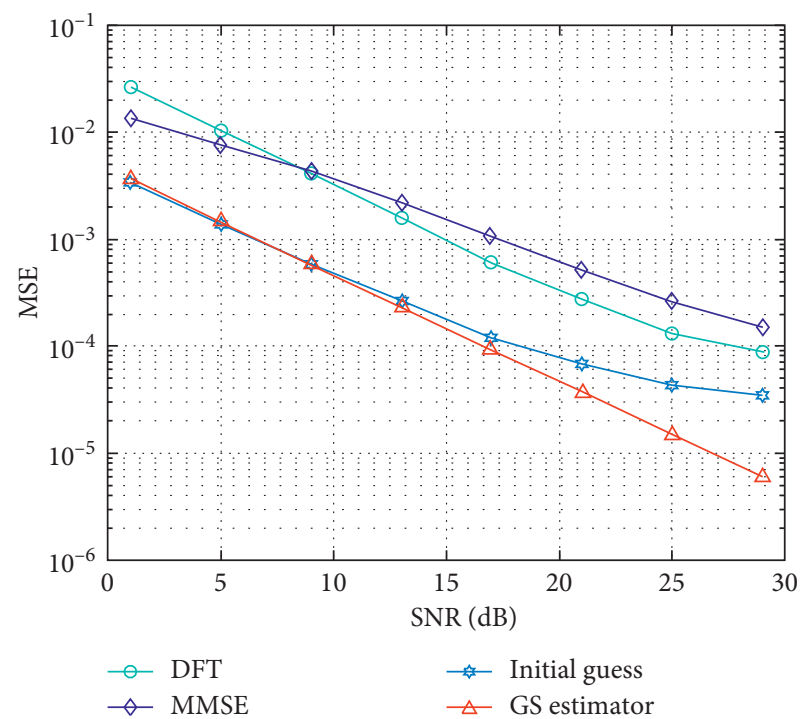

FIgURE 8: Performance of the estimator in GMM channel with $\varnothing=0.01, T=100,16$-QAM.

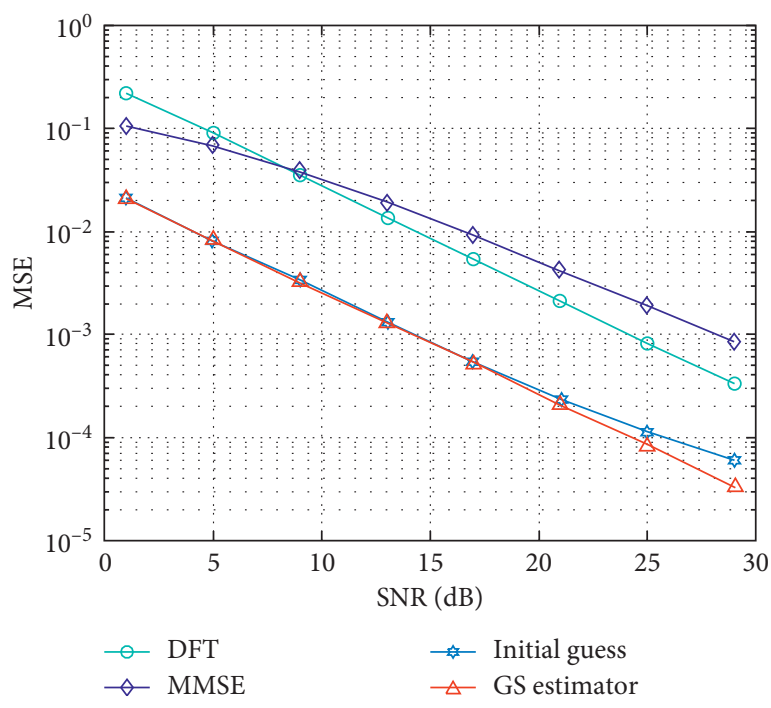

FIGURE 9: Performance of the estimator in GMM channel with $\varnothing=0.05, T=100,16-\mathrm{QAM}$.

respectively. In both figures, the numbers of pilot subcarrier vary from 32 to 128 . It can be seen that the MSE improves when the number of pilot subcarrier increases; however, spectral efficiency deteriorates from $98.4 \%$ to $93.8 \%$ with the unity boost factor of the pilot subcarrier. It is also observed 


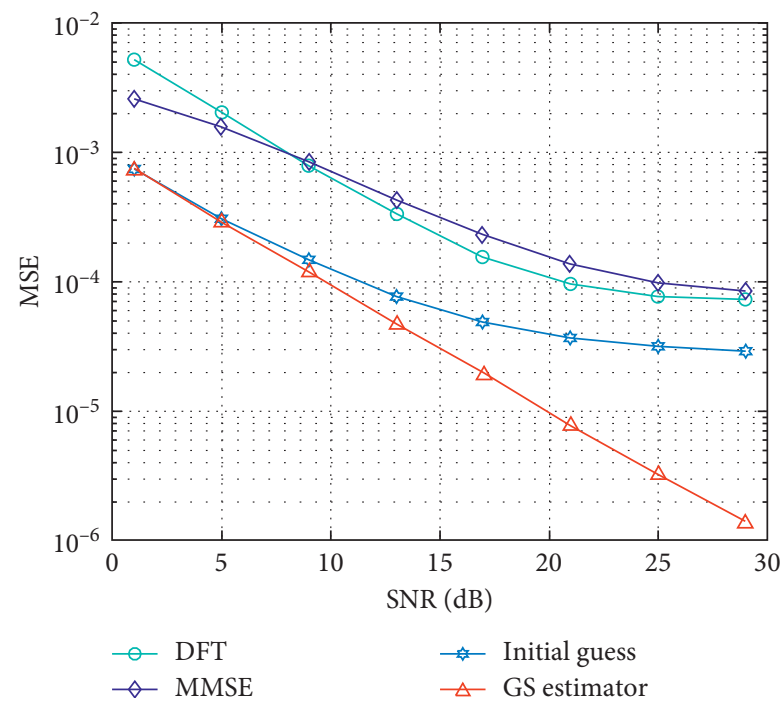

FIgure 10: Performance of the estimator in GMM channel with $\varnothing=0.01, T=100,4$-QAM.

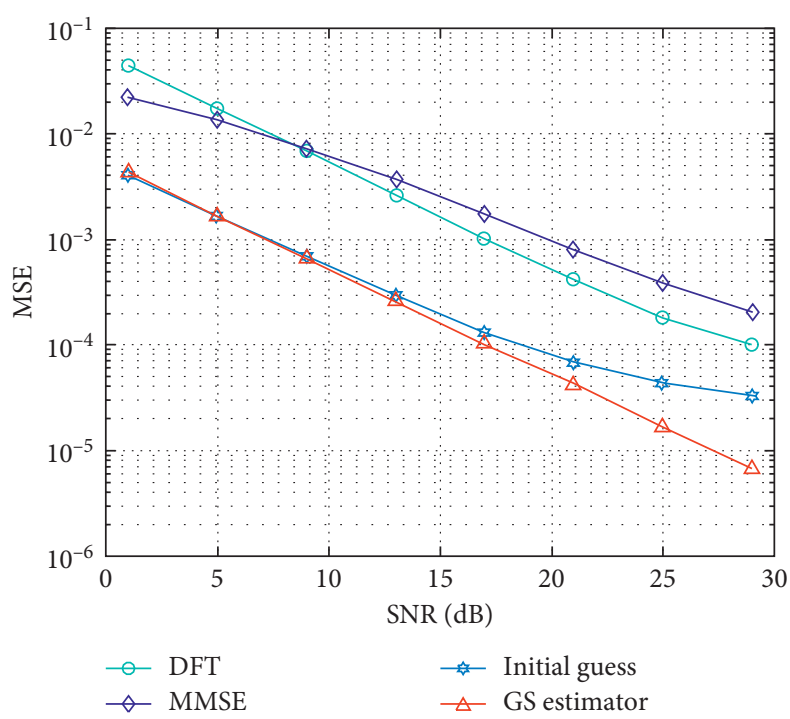

FIgURE 11: Performance of the estimator in GMM channel with $\varnothing=0.05, T=100,4$-QAM.

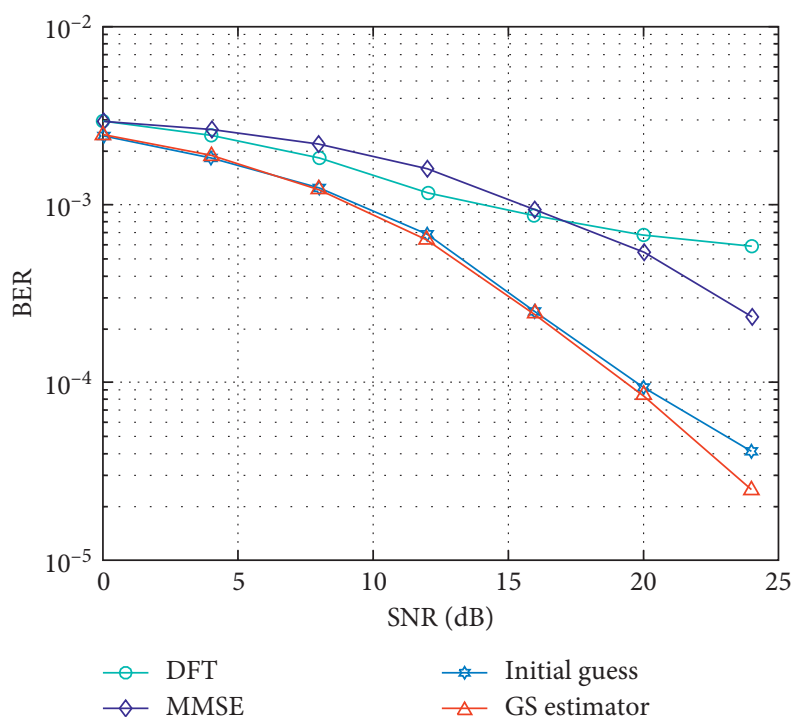

FIGURE 12: BER performance of the estimator in an AWGN channel with $\varnothing=0, T=0$ and 16-QAM, code rate $=1 / 2$. 


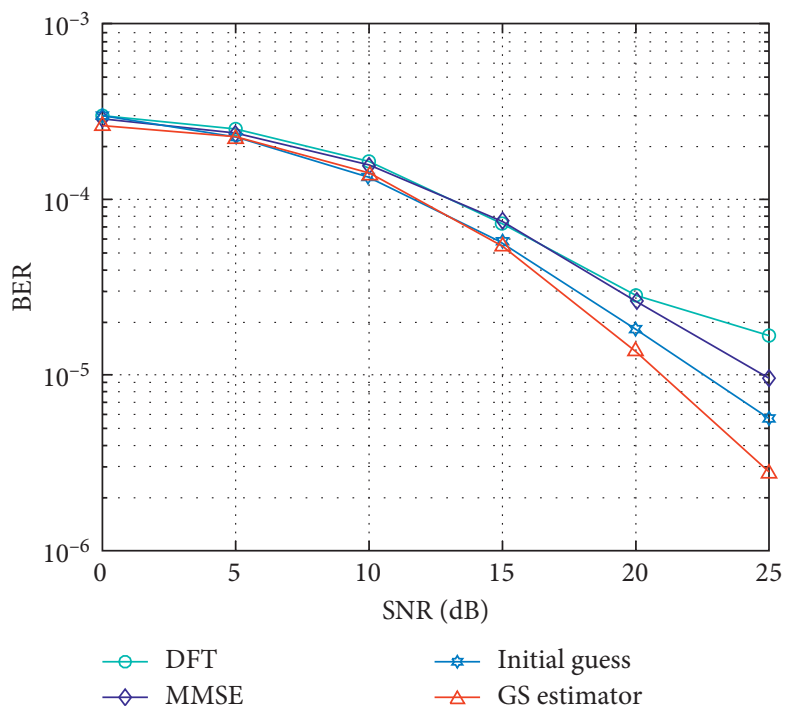

FIGURE 13: BER performance of the estimator in GMM channel with $\varnothing=0.01, T=100$ and code rate $=1 / 2$.

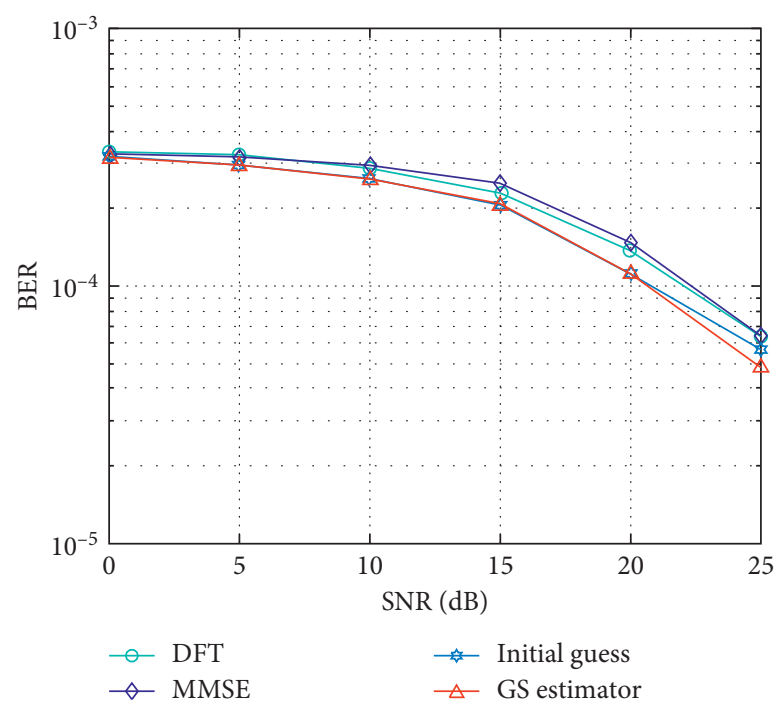

FIGURE 14: BER performance of the estimator in GMM channel with $\varnothing=0.05, T=100$ and 16-QAM, code rate $=1 / 2$.

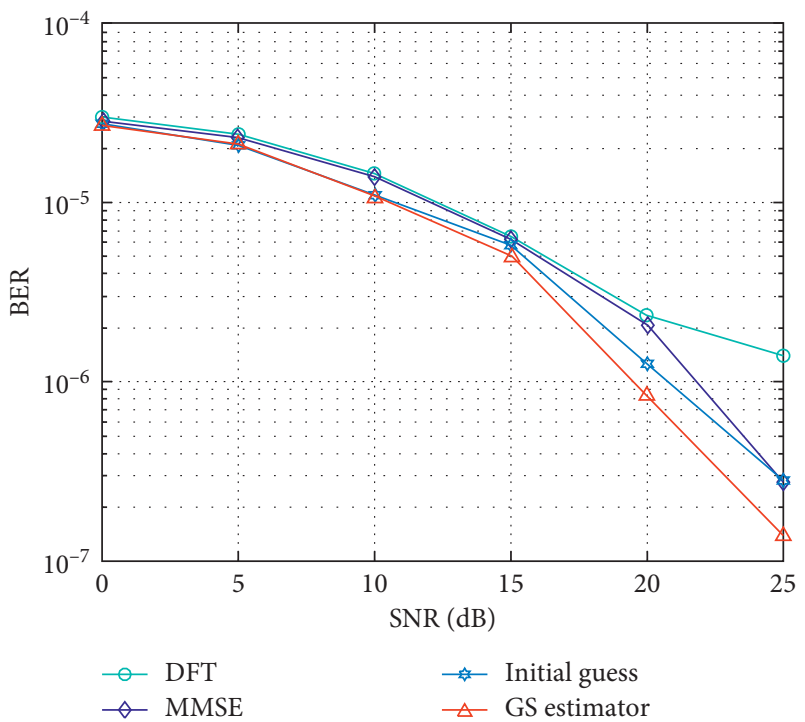

FIGURE 15: BER performance of the estimator in GMM channel with $\varnothing=0.01, T=100$ and code rate $=1 / 3$. 


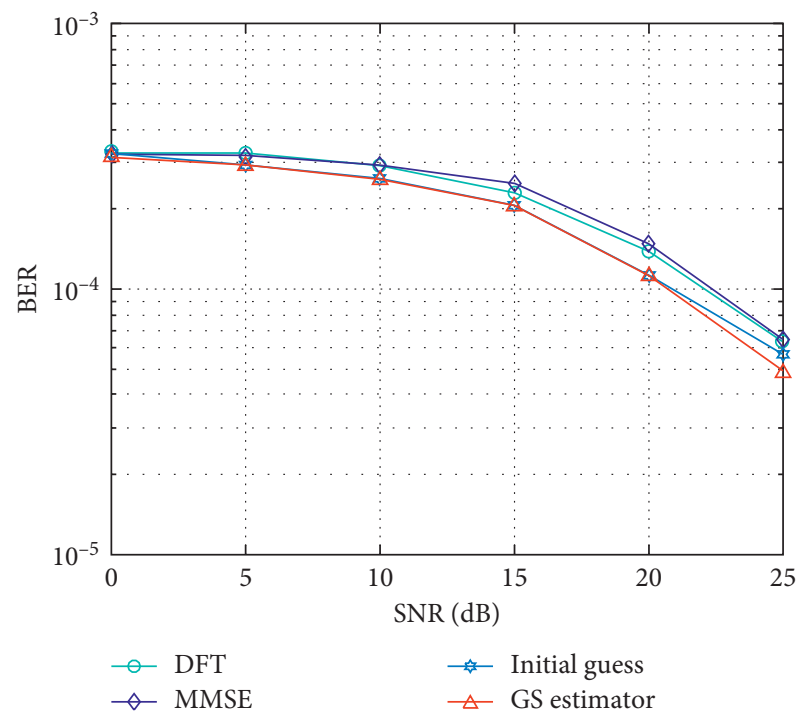

FIGURE 16: BER performance of the estimator in GMM channel with $\varnothing=0.05, T=100$ and code rate $=1 / 3$.

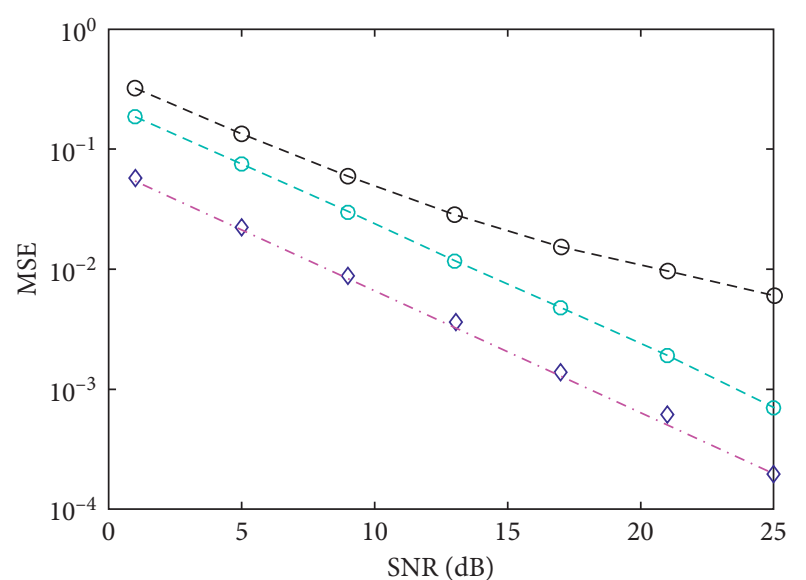

$$
\begin{aligned}
-\ominus-\text { No. of pilots } & =32, \text { boosting factor }=1, \eta=98.4 \% \\
-\ominus-\text { No. of pilots } & =64, \text { boosting factor }=1, \eta=96.8 \% \\
\diamond \quad \text { No. of pilots } & =128, \text { boosting factor }=1, \eta=93.8 \% \\
-- \text { No. of pilots } & =64, \text { boosting factor }=16 / 9, \eta=96.8 \%
\end{aligned}
$$

FIGURE 17: Effect of boosting factor of pilot subcarrier for the proposed time-domain estimator.

that the minimum MSE is achieved when 128 pilot subcarriers are used. In Figure 17, it can be seen that a similar MSE and higher spectral efficiency can be achieved when 16/ 9 boosted power level of pilot subcarriers is used with 64 pilot subcarriers for the proposed estimator. However, the impact of the boosting factor of pilot subcarriers is not prominent in frequency-domain channel estimator, especially at the high SNR regime in Figure 18. The performance of frequency-domain estimators mainly depends upon interpolation, which decreases with the density of the pilots. Hence, the impact of the boosting factor of the pilot subcarriers can be achieved in the time-domain channel estimation schemes only.

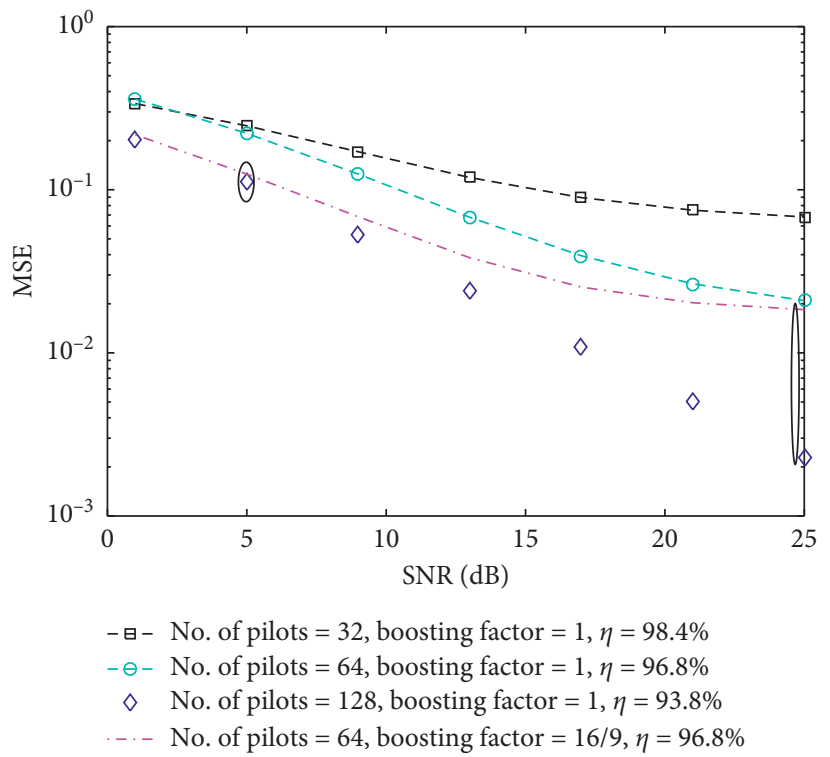

FIGURE 18: Effect of boosting factor of pilot subcarrier for the frequency-domain MMSE estimator.

\section{Conclusion}

The presented work investigates an efficient and low-complexity Gauss-Seidel based time-domain channel estimation method for AWGN and GMM environments, where the Gauss-Seidel method is utilized to obtain a high convergence rate by estimating an appropriate initial value. The simulation results show that the proposed estimator achieves much better accuracy compared to DFT and MMSE algorithms. Moreover, the effect of the boosting factor of the pilot subcarriers is analyzed in the proposed time-domain estimator. The computation complexity of the presented estimator is analyzed on FPGA, which shows the number of hardware recourses' utilization, schematic diagram, and timing diagram. Furthermore, it provides acceptable results for GMM environments, up to the probability of impulsive component of 5\%. Therefore, one would like to extend the presented work by considering higher probabilities of the impulsive noise component. Another possible extension of this work is to investigate the SNR estimation in a similar environment.

\section{Data Availability}

No data were used to support this study.

\section{Conflicts of Interest}

The authors declare that they have no conflicts of interest.

\section{Acknowledgments}

This experimental work was supported by Higher Education Commission (HEC), Pakistan, under Grant 1710/SRGP/ R\&D/HEC for Sir Syed University of Engineering and Technology, Karachi. 


\section{References}

[1] L. Gordon, Stuber, "Principles of Mobile Communication", Kluwer Academic Publication, New York, NY, USA, 2nd edition, 2002.

[2] T. Zhang and G. Gui, "Sign function based sparse adaptive filtering algorithms for robust channel estimation under nonGaussian noise environments," Algorithms, vol. 9, no. 3, p. 54, 2016.

[3] A. M. Khan and V. Jeoti, "On time-domain OFDM channel estimation: use of pilots correlation for digital video broadcasting (DVB) cable receiver," Optoelectronics and Advanced Materials- Rapid Communications, vol. 9, no. 5, pp. 891-897, 2015.

[4] Y. Wang, Z. Zhang, and Y. Chen, "Pilot-aided joint channel estimation for OFDM based cooperative multi-cell networks," Telkomnika Indonesian Journal of Electrical Engineering, vol. 12, pp. 6860-6867, 2014.

[5] R. J. Kozick and B. M. Sadler, "Maximum-likelihood array processing in non-Gaussian noise with Gaussian mixtures," IEEE Transactions on Signal Processing, vol. 48, no. 12, pp. 3520-3535, 2000.

[6] D. Middleton, "Non-Gaussian noise models in signal processing for telecommunications: new methods an results for class A and class B noise models," IEEE Transactions on Information Theory, vol. 45, no. 4, pp. 1129-1149, 1999.

[7] G. Gui, Li Xu, W. Ma, and B. Chen, "Robust adaptive sparse channel estimation in the presence of impulsive noises," in Proceedings of the 2015, IEEE International Conference on Digital Signal Processing (DSP), pp. 628-632, Singapore, Singapore, July 2015.

[8] E. Hari Krishna, K. Sivani, and K. Ashoka Reddy, "OFDM channel estimation using novel LMS adaptive algorithm," in Proceedings of the 2017 International Conference on Computer, Communication and Signal Processing (ICCCSP), January 2017, Chennai, India.

[9] D. N. Bhange and C. G. Dethe, "Performance analysis of channel estimation techniques for proposed pilot aided 3DOFDM DVB-T2 system," International Journal of Computer Science and Information Security, vol. 14, no. 9, 2016.

[10] M. Aparna, P. V. Vineetha, and S. Kirthiga, "Channel modeling and estimation of polarized mimo for land mobile satellite systems," in Proceedings of the 2017 International Conference on Advances in Computing, Communications and Informatics (ICACCI), Udupi, India, September 2017.

[11] L. Polak and T. Kratochvil, "Exploring of the DVB-T/T2 performance in advanced mobile tv fading channels," in Proceedings of the 2013 36th International Conference on Telecommunications and Signal Processing (TSP), Rome, Italy, July 2013.

[12] D. A. Samo, M. Slimani, G. Baruffa, and L. Rugini, "A performance study of DVB-T2 and DVB-T2-Lite for mobile reception," Digital Signal Processing, vol. 37, pp. 35-42, 2015.

[13] A. T. Muharrem and E. Perrins, "Pilot based channel estimation and transform domain analysis in broadband PLC for smart grid," in Proceedings of the 2013 IEEE International Conference on Smart Grid Communications (SmartGridComm), pp. 283-288, Vancouver, Canada, October 2013.

[14] Y. Zhao and A. Huang, "A novel channel estimation method for ofdm mobile communication systems based on pilot signals and transform-domain processing," in Proceedings of the 1997 IEEE 47th Vehicular Technology Conference Technology in Motion, pp. 2089-2093, Phoenix, AZ, USA, May 1997.
[15] M. Zourob and R. Rao, " $2 \times 1-D$ fast fourier transform interpolation for lte-a ofdm pilot-based channel estimation," in Proceedings of the 2017 International Conference on Electrical and Computing Technologies and Applications (ICECTA), Ras Al Khaimah, UAE, November 2017.

[16] H.-M. Wang, J.-C. Jiang, and Y.-N. Wang, "Model refinement learning and an example on channel estimation with universal noise model," IEEE Journal on Selected Areas in Communications, vol. 39, no. 1, pp. 31-46, 2021.

[17] Y. Qiang, X. Shao, and X. Chen, “A model-driven deep learning algorithm for joint activity detection and channel estimation," IEEE Communications Letters, vol. 24, no. 11, pp. 2508-2512, 2020.

[18] V. U. Trung Kien, K. H. Manh, and L. E. Hung Lan, "Performance enhancement of wi-fi fingerprinting-based ips by accurate parameter estimation of censored and dropped data," Radio Engineering, vol. 28, no. 4, pp. 740-748, 2019.

[19] C.-K. Wen, S. Jin, K.-K. Wong, J.-C. Chen, and P. Ting, "Channel estimation for massive MIMO using Gaussianmixture bayesian learning," IEEE Transactions on Wireless Communications, vol. 20, pp. 1356-1368, 2014.

[20] J. Lin, M. Nassar, B. L. Evans, and B. L. Evans, "Impulsive noise mitigation in powerline communications using sparse bayesian learning," IEEE Journal on Selected Areas in Communications, vol. 31, no. 7, pp. 1172-1183, 2013.

[21] T. Zhang and G. Gui, "Reweighted factor selection for SLMSRL1 algorithm under Gaussian mixture noise environments," Algorithms, vol. 8, no. 4, pp. 799-809, 2015.

[22] G. Gui, Li Xu, and N. Shimoi, "Stable sparse channel estimation algorithm under non-Gaussian noise environments," in Proceedings of the 2015 21st Asia-Pacific Conference on Communications (APCC), pp. 561-565, Kyoto, Japan, October 2015.

[23] W. Kim, H. John, and L. Hansen, “Advanced parallel gaussian mixture model based feature compensation integrated with iterative channel estimation," Speech Communication, vol. 73, pp. 81-93, 2015.

[24] R. Liu, T.-L. Kung, and K. K. Parhi, "Impulse noise correction in OFDM systems," Journal of Signal Processing Systems, vol. 74, no. 2, pp. 245-262, 2014.

[25] Z.-Q. He, H. Li, Z.-P. Shi, J. Fang, and L. Huang, "A robust iteratively reweighted 12 approach for spectral compressed sensing in impulsive noise," IEEE Transactions on Broadcasting, vol. 46, pp. 215-220, 2017.

[26] K. Ogura, A. Nakamura, and M. Itami, "A study on class an impulsive noise cancellation and channel estimation under Rayleigh fading environment," in Proceedings of the 2018, IEEE International Conference on Consumer Electronics (ICCE), pp. 1-2, Las Vegas, NV, USA, January 2018.

[27] K. Yan, H. Zhang, and H.-C. Wu, "Robust multipath channel estimation in the presence of impulsive noise," IET Communications, vol. 12, no. 2, pp. 228-235, 2018.

[28] B. Partap and P. Singh, "Hybrid approach for channel estimation using iterative compensation and LMS algorithm," International Journal of Engineering \& Technology, vol. 7, no. 3, pp. 34-37, 2018.

[29] L. Mingqi, T. Jianguo, and Z. Wenjun, "A channel estimation method based on frequency-domain pilots and time-domain processing for OFDM systems," IEEE Transactions on Consumer Electronics, vol. 50, pp. 1049-1057, 2004.

[30] W. Luo, Q. Ou, Y. Fei, L. Cui, and J. Jin, "Analysis of a new hidden attractor coupled chaotic system and application of its weak signal detection," Mathematical Problems in Engineering, vol. 2020, Article ID 8849283, 15 pages, 2020. 
[31] ETSI TS 102831 V1.2.1 (2012-08), Digital Video Broadcasting (DVB); Implementation Guidelines for a Second Generation Digital Terrestrial Television Broadcasting System (DVB-T2), ETSI, Cannes, French, 2012.

[32] R. Wang, C. Li, S. Çiçek, K. Rajagopal, and X. Zhang, "A memristive hyperjerk chaotic system: amplitude control, FPGA design, and prediction with artificial neural network," Complexity, vol. 2021, Article ID 6636813, 17 pages, 2021.

[33] N. Wu, A. Zain Anwar, M. Mujtaba Shaikh, M. Rehan Yahya, and M. Aamir, "Compact and high speed architectures of KASUMI block cipher," Wireless Personal Communications, vol. 106, no. 4, pp. 1787-1800, 2019. 